\title{
Immediate results of complex treatment of patients with generalized periodontitis of extraordinary development with a predominance of the parasympathetic nervous system
}

\author{
Viktor M. Batig ${ }^{1}$, Anatoly V. Borysenko ${ }^{2}$, Tetiana A. Hlushchenko ${ }^{1}$, Irina V. Batig ${ }^{1}$, \\ Marianna 0. Ostafiichuk ${ }^{1}$, Olha M. Tokar ${ }^{1}$, Natalia V. Vatamaniuk ${ }^{1}$, Olesia V. Ivanitska ${ }^{1}$, \\ Yuliia H. Kilmukhametova', Michael I. Sheremet ${ }^{3}$, Mikola 0. Ishkov' ${ }^{1}$ \\ ${ }^{1}$ Department of Therapeutic Stomatology, Bukovinian State Medical University, Chernovtsy, Ukraine \\ ${ }^{2}$ Department of Therapeutic Stomatology, O.O. Bohomolets National Medical University, \\ Kyiv, Ukraine \\ ${ }^{3}$ Surgery Department No1, Bukovinian State Medical University, Chernovtsy, Ukraine
}

\begin{abstract}
Topicality. The clinic of generalized periodontitis is caused by a variety of manifestations of the disease in the complex anatomical and physiological complex of periodontal tissues and the general condition of the organism. Therefore, in the treatment of generalized periodontitis, it is necessary to take into account the individual features of the general and periodontal status of the patient.

Aim of the study. Improving the effectiveness of treatment of patients with generalized periodontitis of acute course with a predominance of the parasympathetic nervous system.

Materials and methods. A comprehensive examination of the condition of periodontal tissues of 60 patients and treatment using the proposed scheme of drug therapy. The comparison group performed similarly to the patients in the main treatment group, but without drug premedication.

Results. It was found that the inclusion of the proposed premedication in the complex therapy of patients with generalized periodontitis exacerbated course could effectively affect the inflammatory process in the tissues of the periodontium. This confirms a decrease in PMA index, an increase in the time of hematoma formation during a vacuum test by Kulazhenko, and an improvement in the level of oral hygiene.

Conclusions. The proposed method of treatment allows to eliminate the manifestations of inflammation and to achieve stabilization of the dystrophic-inflammatory process in the periodontium in a shorter period of treatment. In the near future, an earlier and pronounced normalization of clinical and laboratory parameters characterizing the dystrophic-inflammatory process in the periodontium is noted.
\end{abstract}

Keywords: generalized periodontitis, parasympathetic nervous system

Abbreviations: PMA - papillary-marginal-alveolar index; PI - periodontal index

\section{INTRODUCTION}

One of the priority problems of modern dentistry is the problem of treatment and prevention of periodontal diseases. Widespread prevalence, recurrence rate, increase in lesions in young people, the dependence of the occurrence and nature of the disease on various factors. The close correlation of periodontal tissue diseases and the presence of the patient with somatic pathologies is proved.

The general condition of the patient's body determines certain features of the clinical course of generalized periodontitis in the patient, in particular exacerbated its course. The peculiarities of the clinical course of many diseases depend on the condition of the patient's autonomic system, in par- 
ticular the predominance of the sympathetic or parasympathetic autonomic nervous system. This is especially important in the case of an exacerbated course of generalized periodontitis, since in this case there is a sharp decrease in overall resistance in patients.

Thus, in the emergence and development of dystrophic-inflammatory diseases of the periodontium, their combination with various common somatic diseases occupy a special place. Without these features, periodontal diseases have an unfavorable course and resistance to treatment. Given the close relationship between the vascular and the periodontal nervous systems, the autonomic nervous system plays an integral role.

Achieving the balance of sympatho-parasympathetic ratios (by increasing sympathetic activity in patients with parasympathicotonic type) improves blood circulation. Therefore, in the treatment of this type of diseases it is necessary to take into account the condition of the autonomic nervous system of patients. Vegetative disorders are one of the most important intermediate links that realize the pathogenic impact of chronic psycho-emotional stress.

The problem of the participation of the autonomic nervous system in the emergence and development of a number of dental diseases is not given due attention. Therefore, it is important to determine its role in the emergence, development and treatment of dental diseases.

\section{THE AIM OF THE STUDY}

Improving the effectiveness of treatment of patients with generalized periodontitis of acute course with predominance of the parasympathetic nervous system.

\section{MATERIALS AND METHODS}

For the complex treatment of patients with generalized periodontitis with exacerbated disease course, 60 patients with exacerbated course of generalized periodontitis of II-II degree and predominance of parasympathetic nervous system, aged 2545 years were selected. Among them, there were 36 $(71.67 \%)$ women and $24(28.33 \%)$ men. The patients were divided into two groups: the main (40 patients) and the comparison group (20 patients). In both groups, the distribution of patients according to the degree of disease, age and sex was almost the same.

The main study group formed 40 patients. For their treatment, a scheme of drug therapy was developed

For medical preparation for patients with acute course of generalized periodontitis and predominance of the parasympathetic autonomic nervous system, before each visit to the dentist was prescribed:

1. Buskopan $0.01 \mathrm{~g}$ of 1 tablet 3 times a day;

2. Valerian tincture of 0.25 drops 3 times a day.

3. Doxazosin" $0.001 \mathrm{~g}$ per 1 tablet once a day.

After the dental intervention, appoint for 3 days:

1. Ibuprofen $0.2 \mathrm{~g}-2$ tablets 3 times a day;

2. Valerian tincture of 0.20 drops 3 times a day;

3. Buskopan $0.01 \mathrm{~g}$ of 1 tablet 3 times a day;

4. Doxazosin $0.001 \mathrm{~g}$ per 1 tablet once a day.

The assessment of the state of the autonomic nervous system was performed by determining the Kerdo index.

The study did not include patients with severe dentition deformity, malocclusion abnormalities, diseases of the oral mucosa, as well as patients with exacerbations of somatic diseases.

\section{RESULTS AND DISCUSSIONS}

The course of treatment of patients with generalized periodontitis I degree of the main group averaged 7.33 visits, comparison groups - 10.22 visits. To achieve stabilization of the pathological process in patients with stage II generalized periodontitis, it was necessary: 9.67 visits in patients of the main group and 11.75 visits in patients of the comparison group.

After comprehensive treatment, patients in both groups reported improvement in overall well-being, the disappearance of unpleasant sensations and odor from the oral cavity, gum pain and bleeding, discharge from periodontal pockets. Objectively, the mucous membrane of the gums became pale pink in color, compacted, firmly covering the hard tissues of the tooth.

The complex treatment has led to a significant improvement in the hygienic status of patients with generalized periodontitis. This is evidenced by the 
positive dynamics of the OHI-S hygiene index. In patients with I degree of generalized periodontitis of the main group the index of hygiene of OHI-S decreased from $1.86 \pm 0.19$ points to $0.67 \pm 0.07$ points, in patients with II degree of disease the index decreased from $1.98 \pm 0.21$ to $0.77 \pm 0.07$ points. On the whole, in the patients of the main group, the value of the ONI-S index decreased by $62.63 \%$ from $1.93 \pm 0.21$ points to $0.74 \pm 0.08$ points, which corresponds to the level of good oral hygiene.

In patients with grade I generalized periodontitis of the comparison group, the index of hygiene of OHI-S decreased from $1.94 \pm 0.19$ points to $0.88 \pm$ 0.07 points, in patients with grade II disease the index decreased from $1.97 \pm 0.19$ to $0.89 \pm 0.08$ points. In general, in patients in the comparison group, the value of the hygiene index decreased by $55.61 \%$ from $1.96 \pm 0.19$ points to $0.87 \pm 0.08$ points, which also corresponds to the level of good oral hygiene. The data obtained from the values of hygiene indices in the patients of the main group and the comparison group did not differ statistically significantly ( $\mathrm{p}>0.05)$.

The examination showed a significant decrease in pathological mobility of the lower front teeth. In the patients of the main group, the Schiller - Pisarev trial was negative in 12 of $14(85.71 \%)$ patients with grade I generalized periodontitis and in 22 of $26(84.62 \%)$ patients with grade II generalized periodontitis. Overall, the Schiller - Pisarev trial was negative in $34(85.0 \%)$ patients of the main subgroup of patients with generalized periodontitis. In the comparison group, the Schiller Pisarev trial was negative in 5 of $8(62.5 \%)$ patients with grade I generalized periodontitis and 7 of 12 $(58.33 \%)$ patients with grade II generalized periodontitis. Overall, the Schiller - Pisarev trial was negative in $12(60.0 \%)$ patients of the comparison group of patients with generalized periodontitis. The quantitative value of the Schiller-Pisarev test for iodine number of Svrakov was in patients with generalized periodontitis I of the degree of the main subgroup before treatment $2.4 \pm 0.27$ and after treatment $1.3 \pm 0.12(\mathrm{p}<0.05)$. In patients with generalized periodontitis II degree, it was $2.9 \pm 0.29$ before treatment and $1.6 \pm 0.16$ after treatment. Overall, in the main group patients, the numerical value of the Schiller-Pisarev trial was $2.76 \pm 0.27$ before treatment and $1.5 \pm 0.12(\mathrm{p}<0.05)$ after treatment.

The quantitative value of the Schiller-Pisarev iodine test for iodine number of Svrakov was in patients with generalized periodontitis I of the comparison group before treatment $2.3 \pm 0.24$ and after treatment $1.8 \pm 0.18(\mathrm{p}<0.05)$. In patients with generalized periodontitis II degree, it was $2.8 \pm 0.26$ before treatment and after treatment $-1.8 \pm 0.17$. Overall, in the comparison group, it was $2.82 \pm$ 0.27 before treatment and $1.7 \pm 0.12(\mathrm{p}<0.05)$ after treatment. The data obtained were not statistically $(p<0.05)$ different from the data of the main group.

The decrease in gum inflammation was confirmed by the PMA index. In patients of the main subgroup with I degree of the disease its value decreased from $68.3 \pm 2.8 \%$ to $9.22 \pm 0.85 \%$, and in patients with II degree it decreased from $77.3 \pm$ $2.9 \%$ to $12,67 \pm 0.85 \%$. On average, in the main group after treatment, the PMA index decreased by $84.54 \%$ from $74.15 \pm 2.67$ and was $11.46 \pm 0.98 \%$. The comparison group also noted a significant reduction in the level of gum inflammation: the PMA index decreased by $79.59 \%$ from $69.96 \pm 3.77 \%$ to $14.28 \pm 1.31 \%$.

Complex treatment resulted in reduction of gum bleeding, which was evaluated according to the RVI index. In the patients of the main group it decreased by $72.66 \%$ from $2.78 \pm 0.27$ points to 0.76 \pm 0.05 points and in the patients of the comparison group - by $67.02 \%$ from $2.79 \pm 0.22$ points to 0.92 \pm 0.08 points. A significant statistical difference ( $p$ $<0.05)$ was observed between the RVI indices in patients in these groups. These rates were somewhat lower in patients with stage II generalized periodontitis: the RVI decreased 3.73 times in the main subgroup from $2.95 \pm 0.66$ points to $0.79 \pm$ 0.05 points and 3.0 times in the subgroup comparison - from $2.97 \pm 0.36$ points to $0.98 \pm 0.09$ points.

Changes in the periodontal index (PI) testify to the condition of the entire periodontal tissue complex at the stages of treatment. With the development and exacerbation of the dystrophic-inflammatory process in the periodontium, the value of the periodontal index increases sharply to an average of $1.98 \pm 0.18$ points at the 1 st degree of generalized periodontitis and to $3.22 \pm 0.33$ points at the 2nd degree of generalized periodontitis. After comprehensive treatment of patients with generalized 
periodontitis, the index value decreases. In patients with generalized periodontitis of the first degree of the main group, the PI index decreased by $73.13 \%$ from $1.98 \pm 0.18$ points to $0.73 \pm 0.06$, in patients with the second degree of the disease it decreased by $74.85 \%$ from $3,22 \pm 0.33$ to $0.81 \pm 0.08$ points. In general, in the patients of this main group, the PI index decreased by $74.55 \%$ from $2.79 \pm 0.3$ to 0.71 \pm 0.07 points.

In the comparison group, after treatment, the periodontal index decreased in patients with grade I generalized periodontitis from $1.92 \pm 0.64$ points to $0.86 \pm 0.1$ points and in patients with stage II generalized periodontitis decreased from $3.43 \pm$ 0.34 points to $1.17 \pm 0.01$ points. On average, patients in the comparison group had a periodontal index drop of $48.49 \%$ from $2.52 \pm 0.48$ to $1.04 \pm$ 0.07 points.

Depending on the nature and severity of the dystrophic-inflammatory process in periodontal tissues, the number of leukocytes migrating into the oral cavity increases. Their number is largely determined by the state of permeability of the gum capillaries and the exacerbated nature of the course of generalized periodontitis. With the exacerbated course of the pathological process in the periodontium, it reached an average of $478.5 \pm 36.3$ cells per $1 \mathrm{~mm} 3$ of the wash fluid. The percentage of living neutrophilic granulocytes averaged $61.3 \pm 3.46 \%$. Accordingly, the number of cells of the fused epithelium was increased to $287.6 \pm 10.6$.

After complex treatment, there is a decrease in the number of leukocyte emigration into the oral cavity by an average of $156.5 \pm 16.8$ cells per 1 $\mathrm{mm} 3$ of wash fluid. The number of living neutrophilic granulocytes increases to $78.5 \pm 6.7$, respectively, due to the nature of the course of generalized periodontitis. The number of cells of the fused epithelium was reduced according to $107.4 \pm 8.2$ cells in $1 \mathrm{~mm} 3$ of the wash fluid. Thus, there is a significant decrease in the number of leukocytes that migrated into the oral cavity and cells of the fused epithelium, indicating a decrease in the intensity of inflammation in periodontal tissues. In the comparison subgroup, these changes are slightly less pronounced.

In the study of the content of periodontal pockets before treatment revealed a significant amount of diverse microflora and different cellular elements. Basically, the cellular composition was rep- resented by neutrophilic granulocytes at different stages of necrobiosis. There is a significant number of their destroyed forms - an average of $77.4 \%$. Phagocytosis is detected in a relatively small number of neutrophilic granulocytes. Incomplete phagocytosis was often noted, which testified to inhibition of protective processes in periodontal tissues. Lymphocytes, polyblasts, and epithelial cells were found in relatively fewer periodontal pockets.

After treatment in the content of periodontal pockets of patients with generalized periodontitis significantly reduces the number of cellular elements and microflora. The relative number of unmodified neutrophilic granulocytes increases and the number of their destroyed forms decreases. At the same time, the number of phagocytosis increases. The number of polyblasts and epithelial cells increases with the elimination of the inflammatory phenomena of the pathological process. In the comparison group, these changes are less pronounced. Compared with the cytological picture of the content of periodontal pockets after treatment in the comparison group, there is a less pronounced significant decrease in the number of destroyed neutrophil granulocytes, an increase in the number of phagocytosis.

Based on the criteria for the effectiveness of treatment of dystrophic-inflammatory process in the periodontium, the results of treatment of patients with generalized periodontitis of the acute course are established. Overall, after comprehensive treatment, stabilization of the pathological process was achieved in $34(85.0 \%)$ of 40 patients of the main subgroup. Of these, $12(85.71 \%)$ of 14 patients with grade I generalized periodontitis and $22(84.62 \%)$ of 26 patients with grade II generalized periodontitis. In the comparison group, favorable treatment outcomes were achieved in 13 $(65.00 \%)$ of the 20 patients of the main subgroup. Of these, $6(75.0 \%)$ of 8 patients with grade I generalized periodontitis and $7(58.33 \%)$ of 12 patients with grade II generalized periodontitis. Deterioration of periodontal condition, no effect of therapy in patients of both subgroups was not detected.

\section{CONCLUSIONS}

After treatment of patients with generalized periodontitis of acute course with predominance of the 
parasympathetic autonomic nervous system with the use of the proposed medicated premedication, they have a significant improvement in the periodontal condition. The proposed method of treatment allows to eliminate the manifestations of inflammation and to achieve stabilization of the dystrophic-inflammatory process in the periodontium in a shorter period of treatment. In the near term of observation, an earlier and pronounced normalization of clinical and laboratory parameters characterizing the dystrophic-inflammatory pro-

\section{REFERENCES}

1. Kérdö I. Ein aus Daten der Blutzirkulation kalkulierter Index zur Beurteilung der vegetativen Tonuslage. Acta Neurovegetativa. 1966; 29(2):250-68.

2. Kinane DF. Causation and pathogenesis of periodontal disease. Periodontol 2000. 2001;25:8-20.

3. Parma C. Parodontopathien. Leipzig: Barth; 1960. 203 p.

4. Danilevsky NF. Systematics of periodontal diseases. Bulletin of dentistry. 1994;1: 17-21.

5. Danilevsky NF, Borisenko AV. Periodontal diseases. Kiev: Health; 2000. 464 p.

6. Lapach SN, Chubenko AB, Babich PN. Statistical Methods in Biomedical Research Using Excel. Kiev: Morion; 2001. 408 p.

7. Mashchenko IS. Periodontal diseases. Dnepropetrovsk: Circle; 2003. $272 \mathrm{p}$.

8. Kuzmin VYU. Correction of vegetative disorders in cerebrovascular diseases with the use of ozone therapy [abstract]. Moscow; 2013. 26 p.

9. Kulazhenko VI Periodontal disease and its treatment using vacuum. Odessa; 1960. 147 p.

10. Sağlam M, Arslan U, Buket Bozkurt Ş, Hakki SS. Boric acid irrigation as an adjunct to mechanical periodontal therapy in patients with chronic periodontitis: a randomized clinical trial. J Periodontol. 2013; 84(9):1297-308.

11. da Costa LFNP, Amaral CDSF, Barbirato DDS, Leão ATT, Fogacci MF. Chlorhexidine mouthwash as an adjunct to mechanical therapy in chronic periodontitis: A meta-analysis. J Am Dent Assoc. 2017; 148(5):308-18.

12. Assem NZ, Alves MLF, Lopes AB, Gualberto EC Junior, Garcia VG, Theodoro LH. Antibiotic therapy as an adjunct to scaling and root planing in smokers: a systematic review and meta-analysis. Braz Oral Res [Internet]. 2017[cited 2019 Jan 23];31:e67.

13. Rovai ES, Souto ML, Ganhito JA, Holzhausen M, Chambrone L, Pannuti CM. Efficacy of Local Antimicrobials in the Non-Surgical Treatment of Patients With Periodontitis and Diabetes: A Systematic Review. J Periodontol. 2016;87(12):1406-17.

14. Xue D, Zhao Y. Clinical effectiveness of adjunctive antimicrobial photodynamic therapy for residual pockets during supportive periodontal therapy: A systematic review and meta-analysis. Photodiagnosis Photodyn Ther. 2017;17:127-33.

15. Lang NP, Tan WC, Krähenmann MA, Zwahlen M. A systematic review of the effects of full-mouth debridement with and without antiseptics in patients with chronic periodontitis. J Clin Periodontol. 2008; 35(8 Suppl):8-21.

16. Herrera D, Sanz M, Jepsen S, Needleman I, Roldán S. A systematic review on the effect of systemic antimicrobials as an adjunct to scaling and root planing in periodontitis patients. J Clin Periodontol. 2002;29(Suppl 3):136-59.

17. Bonito AJ, Lux L, Lohr KN. Impact of local adjuncts to scaling and root planing in periodontal disease therapy: a systematic review. J Periodontol. 2005;76(8):1227-36

18. Gerber FA, Sahrmann P, Schmidlin OA, Heumann C, Beer JH, Schmidlin PR. Influence of obesity on the outcome of non-surgical cess in the periodontium is noted. The data obtained indicate a pronounced beneficial effect of the use of the proposed premedication in the treatment of patients with generalized periodontitis of acute course with a predominance of the parasympathetic autonomic nervous system. The analysis of the results of clinical methods of the study immediately after the complex treatment shows the high therapeutic effectiveness of the effect on the periodontal of the proposed drug treatment.

periodontal therapy - a systematic review. BMC Oral Health [Internet] 2016[cited 2019 Jan 25];16(1):90.

19. Wambier LM, de Geus JL, Chibinski AC, Wambier DS, Rego RO, Loguercio $A D$ et al. Intra-pocket anaesthesia and pain during probing, scaling and root planing: A systematic review and meta-analysis. J Clin Periodontol. 2016;43(9):754-66.

20. Matarese G, Ramaglia L, Cicciù M, Cordasco G, Isola G. The Effects of Diode Laser Therapy as an Adjunct to Scaling and Root Planing in the Treatment of Aggressive Periodontitis: A 1-Year Randomized Controlled Clinical Trial. Photomed Laser Surg. 2017;35(12):702-9.

21. Pamuk F, Lütfioğlu M, Aydoğdu A, Koyuncuoglu CZ, Cifcibasi E, Badur OS. The effect of low-level laser therapy as an adjunct to non-surgical periodontal treatment on gingival crevicular fluid levels of transforming growth factor-beta 1, tissue plasminogen activator and plasminogen activator inhibitor 1 in smoking and non-smoking chronic periodontitis patients: A split-mouth, randomized control study. J Periodontal Res. 2017;52(5):872-82.

22. Soeroso $Y$, Akase T, Sunarto $H$, Kemal $Y$, Salim R, Octavia M et al. The risk reduction of recurrent periodontal pathogens of local application minocycline $\mathrm{HCl} 2 \%$ gel, used as an adjunct to scaling and root planing for chronic periodontitis treatment. Ther Clin Risk Manag [Internet]. 2017[cited 2019 Jan 23];13:307-14. Mahendra J, Mahendra L, Muthu J, John L, Romanos GE. Clinical effects of subgingivally delivered spirulina gel in chronic periodontitis cases: a placebo controlled clinical trial. J Clin Diagn Res. 2013;7(10):2330-3.

23. Paolantonio M, Dolci M, Perfetti G, Sammartino G, D'Archivio D, Spoto $G$ et al. Effect of a subgingival chlorhexidine chip on the clinical parameters and the levels of alkaline phosphatase activity in gingival crevicular fluid during the non-surgical treatment of periodontitis. J Biol Regul Homeost Agents. 2008;22(1):63-72.

24. Greenstein G, Rethman MP. Advantages and limitations of nonsurgical periodontal therapy in the management of chronic periodontitis. Alpha Omegan. 2000;93(4):34-42.

25. Slots J. Periodontitis: facts, fallacies and the future. Periodontol 2000. 2017;75(1):7-23.

26. Sanz M, Teughels W, Adriaens P, Armitage G, Baehni P, Chapple I, et al. Innovations in non-surgical periodontal therapy: Consensus Report of the Sixth European Workshop on Periodontology. J Clin Periodontol. 2008; 35(8 Suppl):3-7.

27. Finkbeiner RL. The results of 1328 periodontal pockets treated with the argon laser: selective pocket thermolysis. J Clin Laser Med Surg. 1995; 13(4):273-81.

28. Gartenmann SJ, Dörig I, Sahrmann P, Held U, Walter C, Schmidlin PR. Influence of different post-interventional maintenance concepts on periodontal outcomes: an evaluation of three systematic reviews. BMC Oral Health [Internet]. 2016[cited 2019 Jan 25];17(1):19.

29. Gaunt F, Devine M, Pennington M, Vernazza C, Gwynnett E, Steen $\mathrm{N}$, et al. The cost-effectiveness of supportive periodontal care for patients with chronic periodontitis. J Clin Periodontol. 2008; 35(8 Suppl):67-82. 